\title{
Stage IV Laryngeal Cancer AJCC v8
}

National Cancer Institute

\section{Source}

National Cancer Institute. Stage IV Laryngeal Cancer A/CC v8. NCI Thesaurus. Code C133161.

Stage IV includes: IVA: (T4a, N0, M0); (T4a, N1, M0); (T1, N2, M0); (T2, N2, M0); (T3, N2, M0); (T4a, N2, M0); IVB: (Any T, N3, M0); (T4b, Any N, M0); IVC: (Any T, Any N, M1). T4a: Supraglottis: Moderately advanced local disease. Tumor invades through the outer cortex of the thyroid cartilage and/or invades tissues beyond the larynx (e.g., trachea, soft tissues of neck including deep extrinsic muscle of the tongue, strap muscles, thyroid, or esophagus). Glottis: Moderately advanced local disease. Tumor invades through the outer cortex of the thyroid cartilage and/or invades tissues beyond the larynx (e.g., trachea, cricoid cartilage, soft tissues of neck including deep extrinsic muscle of the tongue, strap muscles, thyroid, or esophagus). Subg lottis: Moderately advanced local disease. Tumor invades cricoid or thyroid cartilage and/or invades tissues beyond the larynx (e.g., trachea, soft tissues of neck including deep extrinsic muscles of the tongue, strap muscles, thyroid, or esophagus). T3: Supraglottis: T umor is limited to larynx with vocal cord fixation and/or invades any of the following: postcricoid area, preepiglottic space, paraglottic space, and/or inner cortex of thyroid cartilage. Glottis: Tumor is limited to the larynx with vocal cord fixation and /or invades the paraglottic space, and/or inner cortex of the thyroid cartilage. Subglottis: Tumor is limited to the larynx with vocal cord fixation and/or invades the paraglottic space and/or inner cortex of the thyroid cartilage. T1: Supraglottis: Tumor is limited to one subsite of suprag lottis with normal vocal cord mobility. Glottis: T umor is limited to the vocal cord(s) (may involve anterior or posterior commissure) with normal mobility. Subg lottis: Tumor is limited to the subglottis. T2: Supraglottis: Tumor invades the mucosa of more than one adjacent subsite of supraglottis or glottis or region outside the supraglottis (e.g., mucosa of base of the tongue, vallecula, medial wall of pyriform sinus) without fixation of the larynx. Glottis: Tumor extends to supraglottis and/or subglottis, and/or with impaired vocal cord mobility. Subg lottis: T umor extends to vocal cord(s) with normal or impaired mobility. T4b: Supraglottis: Very advanced local disease. T umor invades prevertebral space, encases carotid artery, or invades mediastinal structures. Glottis: Very advanced local 
disease. Tumor invades prevertebral space, encases carotid artery, or invades mediastinal structures. Subg lottis: Very advanced local disease. Tumor invades prevertebral space, encases carotid artery, or invades mediastinal structures. N0: No regional lymph node metastasis. N1: Metastasis in a single ipsilateral lymph node, $3 \mathrm{~cm}$ or less in greatest dimension and ENE(-). N2: Metastasis in a single ipsilateral lymph node, $3 \mathrm{~cm}$ or less in greatest dimension and $\operatorname{ENE}(+)$; or metastasis in a single ipsilateral lymph node, more than $3 \mathrm{~cm}$ but not more than $6 \mathrm{~cm}$ in greatest dimension and ENE(-); or metastases in multiple ipsilateral lymph nodes, none more than $6 \mathrm{~cm}$ in greatest dimension and $\operatorname{ENE}(-)$; or metastasis in bilateral or contralateral lymph nodes, none more than $6 \mathrm{~cm}$ in greatest dimension and ENE(-). N3: metastasis in a lymph node, more than $6 \mathrm{~cm}$ in greatest dimension and ENE(-); or metastasis in a single ipsilateral lymph node, more than $3 \mathrm{~cm}$ in greatest dimension and $\mathrm{ENE}(+)$; or metastases in multiple ipsilateral, contralateral, or bilateral lymph nodes and any with $\mathrm{ENE}(+)$. M0: No distant metastasis. M1: distant metastasis. (AJCC 8th ed.) 